\title{
Detection of Entorhinal Layer II Using Tesla Magnetic Resonance Imaging
}

Jean C. Augustinack, PhD, ${ }^{1}$ Andre J. W. van der Kouwe, PhD, ${ }^{1}$ Megan L. Blackwell, BS, ${ }^{1,2}$

David H. Salat, PhD, ${ }^{1}$ Christopher J. Wiggins, PhD, ${ }^{1}$ Matthew P. Frosch, MD, PhD, ${ }^{3}$

Graham C. Wiggins, D.Phil, ${ }^{1}$ Andreas Potthast, PhD ${ }^{1}$ Lawrence L. Wald, PhD, ${ }^{1}$ and Bruce R. Fischl, PhD ${ }^{1,2}$

The entorhinal cortex lies in the mediotemporal lobe and has major functional, structural, and clinical significance. The entorhinal cortex has a unique cytoarchitecture with large stellate neurons in layer II that form clusters. The entorhinal cortex receives vast sensory association input, and its major output arises from the layer II and III neurons that form the perforant pathway. Clinically, the neurons in layer II are affected with neurofibrillary tangles, one of the two pathological hallmarks of Alzheimer's disease. We describe detection of the entorhinal layer II islands using magnetic resonance imaging. We scanned human autopsied temporal lobe blocks in a 7T human scanner using a solenoid coil. In 70 and $100 \mu \mathrm{m}$ isotropic data, the entorhinal islands were clearly visible throughout the anterior-posterior extent of entorhinal cortex. Layer II islands were prominent in both the magnetic resonance imaging and corresponding histological sections, showing similar size and shape in two types of data. Area borders and island location based on cytoarchitectural features in the mediotemporal lobe were robustly detected using the magnetic resonance images. Our ex vivo results could break ground for high-resolution in vivo scanning that could ultimately benefit early diagnosis and treatment of neurodegenerative disease.

Ann Neurol 2005;57:489-494

The entorhinal cortex (EC) lies in the mediotemporal lobe and it has major cytoarchitectural, functional, and clinical significance. First, EC layer II contains large stellate and pyramidal neurons that form clusters or islands, making it a unique cytoarchitecture in the human brain. ${ }^{1-4}$ Macroscopically, the islands are observed grossly on the surface of the EC known as verrucae, meaning wartlike, ${ }^{5}$ and can be seen on a Nissl-stained section without a microscope. Second, vast cortical input to the EC arises from visual, auditory, somatic, and limbic association cortices; it is here that incoming sensory information ultimately converges. ${ }^{6,7}$ In turn, the major output of the EC arises from layers II and III and forms the perforant pathway that terminates on the dentate gyrus (DG) granule cells and hippocampal pyramidal neurons. ${ }^{8-12}$ Third and clinically, the neurons in layer II are among the first cells affected with neurofibrillary tangles (NFTs), one of the two pathological hallmarks of Alzheimer's disease. ${ }^{13,14}$ The EC is devastated in Alzheimer's disease, and at end-stage $\mathrm{AD}$ contains a heavy burden of NFTs in all layers. Neuronal loss in entorhinal layer II islands has been shown with Alzheimer's disease, with a $60 \%$ loss in even mild cases. ${ }^{15,16}$ Thus, the layer II islands are structurally, functionally, and clinically significant, and examining the structure of entorhinal layer II using MRI would be of great potential utility for all these domains. In this study, we used a human whole-body $7 \mathrm{~T}$ scanner, obtaining images with $100 \mu \mathrm{m}$ isotropic voxels, and were able to robustly image the entorhinal islands, which had not been observed previously using MRI.

\section{Subjects and Methods}

Four healthy control brain hemisphere samples were obtained from the Massachusetts General Hospital Autopsy Service and were fixed at autopsy with $10 \%$ formalin for at least 2 weeks (mean age, 56 years; three men, 1 woman; postmortem interval before fixation, $<24$ hours). Anterior and medial temporal lobe blocks were dissected and placed in heptacosafluorotributylamine (Sigma, St. Louis, MO) to minimize background effects.

Images were collected on a 7T whole-body MRI scanner based on a Siemens Sonata platform (Siemens Medical Solu-
From the ${ }^{1}$ Department of Radiology, Athinoula A. Martinos Center for Biomedical Imaging, Massachusetts General Hospital, Charlestown, MA; ${ }^{2} \mathrm{MIT} / \mathrm{HST}$, Massachusetts Institute of Technology, Cambridge, MA; ${ }^{3}$ Department of Neuropathology, Massachusetts General Hospital, Charlestown, MA.

Received Sep 15, 2004, and in revised form Dec 13. Accepted for publication Jan 11, 2005.
Published online Mar 28, 2005, in Wiley InterScience (www.interscience.wiley.com). DOI: 10.1002/ana.20426

Address correspondence to Dr Augustinack, Massachusetts General Hospital-CNY, 149 13th St., Room 2248, Charlestown MA 02129. E-mail: jean@nmr.mgh.harvard.edu. 
tions, Erlangen, Germany) using a solenoid coil $(28.5 \mathrm{~mm}$ inside diameter $\times 44 \mathrm{~mm}$ in length, 3 turn). A conventional three-dimensional spoiled gradient-echo sequence was used with a resolution of 70 and $100 \mu \mathrm{m}(70 \mu \mathrm{m}$ isotropic data: field of view, $36 \mathrm{~mm}$; slab thickness, $24.64 \mathrm{~mm}$; repetition time, 23 milliseconds; echo time, 11.2 milliseconds; bandwidth, $92 \mathrm{~Hz}$ per pixel; scan duration, 45 minutes 20 seconds; Fourier encoding was $6 / 8$ in the phase-encoding direction and $7 / 8$ in the partition direction; $100 \mu \mathrm{m}$ isotropic data: field of view, $52 \mathrm{~mm}[512 \times 512$ matrix]; slab thickness, $25.6 \mathrm{~mm}$ [ 256 partitions]; repetition time, 20 milliseconds; echo time, 7.82 milliseconds; bandwidth, $134 \mathrm{~Hz}$ per pixel; scan duration, 43 minutes 41 seconds; full Fourier encoding). A total of 18 scans were acquired with varying flip angles (3 scans at each of the following angles: 20, 15, 10, 5 , 3 , and 1 degree). For the $100 \mu \mathrm{m}$ scans, fast, low-angle shot images were acquired with a fixed echo time $(7.8$ milliseconds) and repetition time (20 milliseconds), with flip angles that varied from 5 to 25 degrees in steps of 5 degrees. These were used to estimate the T1 and proton density of the sample at each point in space. ${ }^{17}$ Notably, this sequence ordinarily would result in a $\mathrm{T} 1$-weighted image; however, fixation-induced shortening of the $\mathrm{T} 1$ of gray and white matter removes almost all $\mathrm{T} 1$ weighting, resulting in a strongly proton density-weighted image. ${ }^{18,19}$ Images then were synthesized at all flip angles from 1 to 90 degrees, and 23 degrees was determined to be the flip angle that maximized the contrast between the entorhinal islands and the surrounding cortex.

After magnetic resonance scanning, tissue blocks were placed in $15 \%$ glycerol for 3 days and sectioned at $50 \mu \mathrm{m}$ on a freezing sliding microtome. Sections were mounted on gelsubbed slides, pretreated and defatted before being stained with buffered thionin, differentiated and dehydrated through a series of alcohols, and cleared in xylene. The sections were coverslipped with Permount (Fisher Scientific, Fair Lawn, NJ). In the Nissl stain, the layer II entorhinal islands were well stained with no qualitative indication of cell death.

Statistics used in this study were one-way analysis of variance with Fisher's post hoc test. The significance value was set at $p<0.05$.

\section{Results}

In three of four brain samples, the entorhinal islands were clearly visible throughout the anterior-posterior extent of the EC in proton density-weighted images at 70 and $100 \mu \mathrm{m}$ data (Fig 1 ). The EC is bordered anteriorly
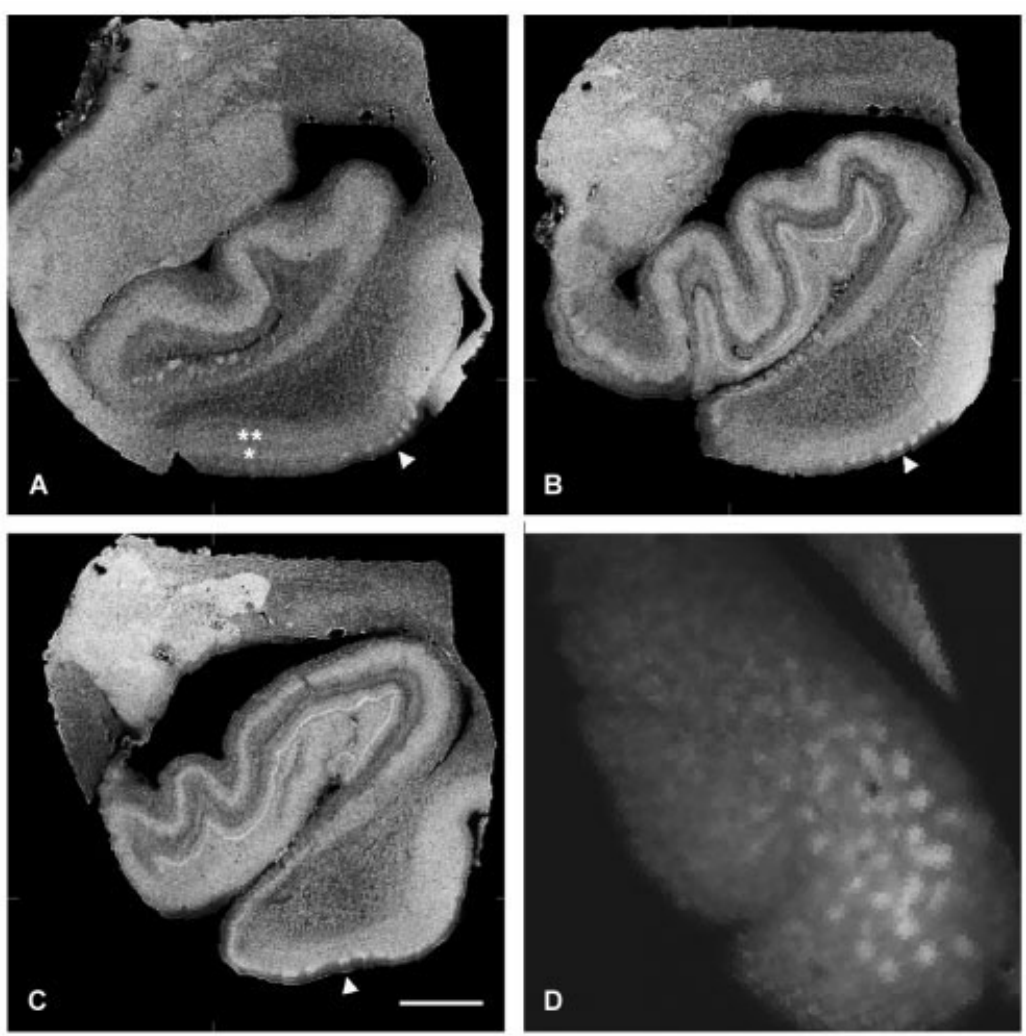

Fig 1. Proton density-weighted images $(100 \mu \mathrm{m})$ of the anterior parahippocampal gyrus (ie, entorhinal cortex [EC]) collected from $7 T$ scanner with a $28.5 \times 44 \mathrm{~mm}$ solenoid coil. Entorhinal islands are shown throughout the rostrocaudal extent of the parahippocampal gyrus at the level of the (A) amygdala, (B) uncal hippocampus, and (C) posterior hippocampus. (A-C) Sections are sliced coronally; (D) section is sliced tangentially. White arrowheads point to the best examples of entorhinal layer II (A-C). (A) Additional layers in the EC are observed: a single asterisk designates layer III, and two asterisks designate layer IV. (D) A digital tangential section of the entorhinal layer II is represented; the bright white areas represent the entorhinal islands, and the surrounding dark areas are the intervening white matter. Scale bar $=1 \mathrm{~cm}$. 
by the olfactory and perirhinal cortices, medially by parasubiculum, laterally by the perirhinal cortex, and posteriorly by the perirhinal and temporooccipital cortices. Anteriorly, at the rostrocaudal level of the gyrus ambiens, the entorhinal islands are observed laterally close to neighboring perirhinal cortex (see Fig 1A). In addition, the lamina dessicans can be observed on these high-resolution images and observed as the dark lamina, between two brighter areas that are designated with asterisks in Figure 1A. These brighter bands that surround the lamina dessicans represent layers III (single asterisk in Fig 1A) and IV (double asterisk in Fig 1A) and are best observed medially in the EC in Figure 1A. At uncal hippocampal levels of the EC, the entorhinal islands are observed at regular intervals (see Fig 1B). At this level ( $\mathrm{n}=3$ cases), we measured the width of the islands in randomly selected Nissl-stained sections and MRI images at approximately the same rostrocaudal level and found that the mean width $(\mathrm{n}=53$ and $\mathrm{n}=72)$ was $543.0 \pm 146.7$ and $559.7 \pm 108.4 \mu \mathrm{m}$ for Nissl stains and MRI, respectively. All measurements were taken parallel to the pial surface. The Nissl stain and MRI island widths were not significantly different from each other, nor were they significantly different from published values, ${ }^{20}$ suggesting that our MRI data parallel histological data. More posteriorly, entorhinal islands were observed (see Fig 1C), and their slightly different sizes and shapes were commonly observed in human histological preparations. In Figure 1D, the MR image was sliced as a tangential section, with the bright areas in the image representing the entorhinal islands, and the gray areas representing the white matter that surrounds them, known as the reticular substance of Arnold ${ }^{21}$ or entorhinal layer II interislands.

To validate the entorhinal islands observed in the MRI data, we used histological methods. After MRI scanning, we sectioned and stained with thionin, a
Nissl stain, to detect the presence of entorhinal islands. In three of four cases, the entorhinal islands observed in the MRI (Fig 2A) corresponded to the entorhinal islands in the Nissl-stained section (see Fig 2B). In one case, the MRI results did not clearly distinguish islands, whereas the corresponding Nissl-stained section showed entorhinal layer II islands. Nonetheless, using the histology section as a guide in this case, the entorhinal islands were faintly visible, and the lamina dessicans was clearly discernible.

Strictly from a mediolateral perspective, the EC is situated between the parasubiculum and perirhinal cortex. Between these two areas, the layer II islands were prominent, and the EC showed a different MRI pattern than the surrounding tissue. The EC, parasubiculum, presubiculum, and subiculum were labeled manually with perpendicular lines drawn from the gray/white border to the pial surface. Each line defined an intensity profile that represents the laminar distribution of MRI values throughout the cortical layers (using the 23-degree flip angle $100 \mu \mathrm{m}$ isotropic synthesized data). The norm of the difference between adjacent profiles was computed, and prominent peaks were observed at the junction of each of the four manually labeled cortical areas (EC, parasubiculum, presubiculum, and subiculum) with its neighbor, indicating the ability to distinguish cytoarchitectural boundaries with MRI (Fig 3).

To answer the question whether the contrast we observe in the MRI is, in fact, the islands and not reticular substance of Arnold (interisland EC layer II), we measured the pixel intensity or luminosity $(\mathrm{n}=3)$ using Adobe Photoshop (Adobe Systems, Mountain View, CA) for EC layer II islands and EC layer II interislands, as well as other known white and gray matter structures, alveus (white), angular bundle (white), EC interisland layer II (white), EC layer II (gray), and DG cells (gray). The EC layer II and DG cells (both gray matter) were



Fig 2. Corresponding high-resolution magnetic resonance image (MRI) and histological Nissl section of the entorhinal cortex. (A) A high-resolution $(100 \mu \mathrm{m})$ ex vivo MRI of a 66-year-old man; (B) the corresponding Nissl section stained with thionin. Note the islands in layer II in both types of images (arrowheads) match each other and are demarcated with three black arrowheads. Scale bar $=1 \mathrm{~cm}$. 


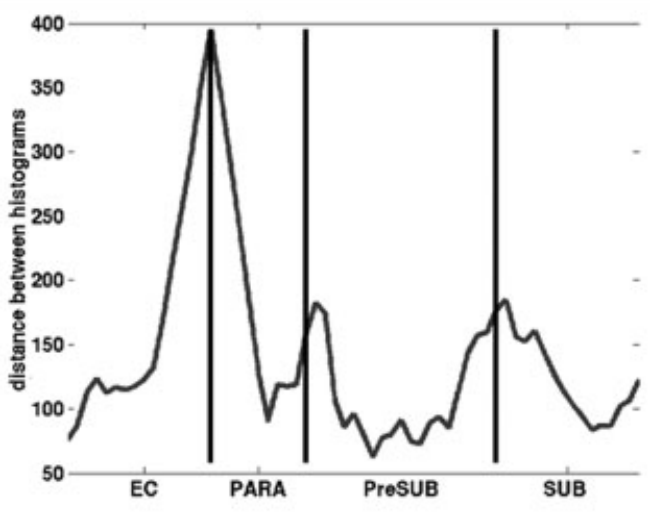

Fig 3. Neighboring cortical areas are predicted with magnetic resonance imaging (MRI) scans across parahippocampal gyrus. Areas including and bordering the entorhinal cortex (EC) were labeled manually with line drawings that originated in the white matter and ended in the gray matter. Note the manual line drawings correlate with the MRI intensity, and labels coincide with the MRI peaks. PARA = parasubiculum; PreSUB = presubiculum; $S U B=$ subiculum.

significantly different from the alveus, angular bundle, and EC layer II interisland (white matter) $(p<0.001)$.

Finally, for one case, the data were split into two groups, and two volumes were created: test (red) and retest (blue) (Fig 4). Manual labels were drawn on the two separate data files and compared. The colocalization (green) between the two volumes, test (red) and retest (blue) shows where they overlap (see Fig 4), indicates the stability of the island location.

\section{Discussion}

Using fast, low-angle shot scans (FLASH) at 7T, we were able to obtain isotropic $100 \mu \mathrm{m}$ resolution and distinguish the entorhinal islands from other cytoarchitectural features in the mediotemporal lobe that until now have not been resolved with other MRI studies using lower magnetic field strengths, larger coils, and different scanning protocols. ${ }^{20,22,23}$ Our ex vivo, high-resolution, fast, low-angle shot scans at 7T showed alternating lowand high-signal intensity in entorhinal layer II. This result suggests that we are able to image the cytoarchitecture of the entorhinal islands that notably are highly metabolic, integrally connected with other brain areas, and vulnerable to disease. ${ }^{24-26}$

Using the same mediotemporal lobe block in each case, we scanned ex vivo tissue using MRI, and then histologically sectioned the block to compare the islands in both datasets. Qualitatively, the entorhinal islands in the MRI are present in the same layer and correspond to islands in the Nissl-stained section. Furthermore, we evaluated and correlated the entorhinal islands in MRI with entorhinal islands in the same Nissl section and established histological and MRI parameters in two ways. First, we measured the EC island width in both MRI and Nissl sections and showed that our MRI EC data width agree not only with the Nissl-stained sections, but also with published values obtained from human entorhinal Nissl sections. ${ }^{20}$ No significant difference was found among the EC widths in the MRI, corresponding Nissl, or published values. In another study comparing MRI and histology, MRI and histological samples were examined in the mediotemporal lobe and showed that MRI volumes correlated with histological volumes. ${ }^{27}$ For our second parameter, we measured the pixel intensity of the islands and compared them with other known white and gray matter structures. We found that the gray matter structures, EC layer II island and DG cells, were not significantly different from each other; however, pixel intensities in gray matter structures were significantly different from those in white matter structures, angular bundle, alveus, and EC layer II interisland. Taken together, these results suggest qualitatively and quantitatively that the entorhinal islands observed in our $7 \mathrm{~T}$ scans are, in fact, real and correlate to each respective Nissl counterpart.

In this study, three of four postmortem cases showed islands in the MRI and corresponding Nissl section. The MRI islands in the fourth case were questionable; however, islands were present in the Nissl-stained sections. Yet, guided by the corresponding Nissl-stained sections in this case, occasional alternating bands were distinguished, and a distinct lamina dessicans was observed throughout the cortex. Also, notably, this case contained an unusual hippocampus that appears to be mal-rotated, but no unusual clinical or pathological information was reported. The lack of islands in this MRI case could be because of unknown tissue properties that are case specific. Because identical scanning protocols were always used and one case did not exhibit islands, each case might require optimization for its particular tissue properties. The amount of fixation time in formalin is variable, but a direct and obvious correlation was not observed, because for the three MRI cases that showed islands, one was fixed for 1 year and the other two were fixed for 2 weeks before scanning. It is clear that further investigation on this case is necessary.

Finally, we evaluated the colocalization of islands when one dataset was split into two volumes and compared. We found that the entorhinal islands in the two volumes colocalized when tested against each other, suggesting that the islands are true neuroanatomical features and are not caused by image noise.

Until now, higher field MRI ex vivo scanning has been largely limited to mouse models ${ }^{28-30}$ or nonhuman primates, ${ }^{31}$ with one exception that examined amyloid-burdened tissue in Alzheimer's disease with T2* analysis. ${ }^{32}$ Other studies have examined ex vivo tissue at lower field strength and have not generated cytoarchitectural MRI data that can be related to histological features; instead, these studies have generated 




Fig 4. The magnetic resonance imaging data were split into two groups, and two volumes were created: one test (red) and the other retest (blue). Manual labels were drawn on two separate data files and compared. The colocalization between the two volumes (green) represents where they overlap.

human brain mapping atlases 33,34 or neuropathological data. ${ }^{35}$ Our $100 \mu \mathrm{m}$ isotropic MRI data not only can achieve cytoarchitecturally defined area borders and pixel intensity profiles, but also can be used to create atlases. Thus, using various parameters, our highresolution data offer the capability for several lines of future computational neuroscience.

MRI assessment of the human brain in the clinical setting has wide-ranging advantages because it is noninvasive, three-dimensional, and highly accessible. Currently, in vivo MRI facilitates the diagnosis of stroke, cancer, and neurodegenerative diseases. There is a growing body of literature examining neurological disease and MRI properties including frontotemporal dementia, ${ }^{36}$ autism, ${ }^{37}$ multiple sclerosis, ${ }^{38-40}$ and Alzheimer's disease. $^{32,41}$ The high-field, high-resolution MRI data presented here showed entorhinal islands that were confirmed by examining corresponding Nissl-stained sections in healthy control human brains. In Alzheimer's disease and temporal lobe epilepsy, whereas current MRI usefulness and utility has been restricted to volumetric assessment with lower field strengths and not cytoarchitectural features, ${ }^{41-46}$ our findings motivate the use of MRI as a tool for whole-brain neuropathology ex vivo. Our results elucidate the type of resolution and signalto-noise ratio needed to extend these techniques to in vivo imaging in healthy and neuropathological brains.

This raises the following question: Is this kind of resolution achievable in vivo? Assuming the average width of entorhinal layer II islands is $\approx 500 \mu \mathrm{m}$, the Nyquist Theorem ${ }^{47}$ indicates that the maximum resolution that can be used in their detection is on the order of $250 \mu \mathrm{m}$ isotropic. To be conservative, we assume that the required resolution is $200 \mu \mathrm{m}$ isotropic. To obtain $200 \mu \mathrm{m}$ resolution in vivo clinically, it will be necessary to use a combination of several techniques including higher field strength, specialized radiofrequency coils, and longer scan time with motion correction. For example, we currently reach resolutions on our 3T Siemens Trio machine (Siemens Medical Solutions) of $400 \mu \mathrm{m}$ isotropic with good signal-to-noise ratio in the mediotemporal lobe using an 8-channel receiver array in a 1-hour scan session. Reducing the voxel size to $200 \mu \mathrm{m}$ requires a signal-to-noise ratio increase of a factor of 8 . First, the increase in field strength to $7 \mathrm{~T}$ results in a decrease in image noise by a factor of 2 . Second, we are currently constructing a 96-channel phased array that we anticipate should yield a further factor of 2, even for deep structures. Finally, recent advances in three-dimensional, real-time motion correction, albeit not in the highresolution context, ${ }^{48,49}$ have yielded the possibility of extremely long scan sessions, and certainly a 2- to 3-hour session is not unreasonable. Thus, as MR technology continues to improve field strength, it is reasonable, although challenging, that we might achieve this type of resolution clinically within the next decade.

This research was supported by the NIH (National Center for Research Resources P41-RR14075, B.R.F.; R01 RR16594-01A1, B.R.F.; and the NCRR BIRN Morphometric Project, BIRN002, B.R.F.), and the Mental Illness and Neuroscience Discovery (MIND) Institute (B.R.F.).

We thank Dr B. Hyman for laboratory resources B.R.F., and A.J. Vidik provide paid consultation for CorTech Labs, Inc.

\section{References}

1. Cajal RS. Studies in the cerebral cortex (translated by Kraft, L.M.) Yearbook, Chicago, 1955;164-179.

2. Lorente de No R. Studies on the structure of the cerebral cortex. Journal Fur Psychologie and Neurologie 1933;45:381-438.

3. Insausti R, Tunon T, Sobreviela $T$, et al. The human entorhinal cortex: a cytoarchitectonic analysis. J Comp Neurol 1995; 355:171-198.

4. Solodkin A, Van Hoesen GW. Entorhinal cortex modules of the human brain. J Comp Neurol 1996;365:610-617.

5. Retzius G. Das Menschenhirn. Stockholm: Norstedt and Sonhe, 1896.

6. Van Hoesen GW, Pandya DN, Butters N. Cortical afferents to the entorhinal cortex of the Rhesus monkey. Science 1972;175: 1471-1473.

7. Jones EG, Powell TP. An anatomical study of converging sensory pathways within the cerebral cortex of the monkey. Brain 1970;93:793-820.

8. Van Hoesen G, Pandya DN, Butters N. Some connections of the entorhinal (area 28) and perirhinal (area 35) cortices of the rhesus monkey. II. Frontal lobe afferents. Brain Res 1975;95:25-38. 
9. Van Hoesen GW, Pandya DN. Some connections of the entorhinal (area 28) and perirhinal (area 35) cortices of the rhesus monkey. III. Efferent connections. Brain Res 1975;95:39-59.

10. Van Hoesen GW. The parahippocampal gyrus: new observations regarding its cortical connections in the monkey. Trends Neurosci 1982;5:345-350.

11. Rosene DL, Van Hoesen GW. Hippocampal efferents reach widespread areas of cerebral cortex and amygdala in the rhesus monkey. Science 1977;198:315-317.

12. Witter MP, Van Hoesen GW, Amaral DG. Topographical organization of the entorhinal projection to the dentate gyrus of the monkey. J Neurosci 1989;9:216-228.

13. Braak H, Braak E. Neuropathological stageing of Alzheimerrelated changes. Acta Neuropathol (Berl) 1991;82:239-259.

14. Hyman BT, Van Hoesen GW, Damasio AR, Barnes CL. Alzheimer's disease: cell-specific pathology isolates the hippocampal formation. Science 1984;225:1168-1170.

15. Gomez-Isla T, Price JL, McKeel DW Jr, et al. Profound loss of layer II entorhinal cortex neurons occurs in very mild Alzheimer's disease. J Neurosci 1996;16:4491-4500.

16. Arriagada PV, Growdon JH, Hedley-Whyte ET, Hyman BT. Neurofibrillary tangles but not senile plaques parallel duration and severity of Alzheimer's disease. Neurology 1992;42:631-639.

17. Fischl B, Salat DH, van der Kouwe A, et al. Sequenceindependent segmentation of magnetic resonance imaging. NeuroImage 2004;23(suppl 1):S69-S84.

18. Pfefferbaum A, Sullivan EV, Adalsteinsson E, et al. Postmortem MR imaging of formalin-fixed human brain. Neuroimage 2004; 21:1585-1595.

19. Tovi M, Ericsson A. Measurements of T1 and T2 over time in formalin-fixed human whole-brain specimens. Acta Radiol 1992;33:400-404.

20. Damasio H, Kuljis RO, Yuh W, et al. Magnetic resonance imaging of human intracortical structure in vivo. Cereb Cortex 1991;1:374-379.

21. Arnold JC. 2Bd vol. Freiburg i. Br.: Herder, 1851.

22. Awad IA, Johnson PC, Spetzler RF, Hodak JA. Incidental subcortical lesions identified on magnetic resonance imaging in the elderly. II. Postmortem pathological correlations. Stroke 1986;17: 1090-1097.

23. Boyko OB, Alston SR, Fuller GN, et al. Utility of postmortem magnetic resonance imaging in clinical neuropathology. Arch Pathol Lab Med 1994;118:219-225.

24. Van Hoesen GW, Solodkin A. Some modular features of temporal cortex in humans as revealed by pathological changes in Alzheimer's disease. Cereb Cortex 1993;3:465-475.

25. Hevner RF, Wong-Riley MT. Entorhinal cortex of the human, monkey, and rat: metabolic map as revealed by cytochrome oxidase. J Comp Neurol 1992;326:451-469.

26. Beall MJ, Lewis DA. Heterogeneity of layer II neurons in human entorhinal cortex. J Comp Neurol 1992;321:241-266.

27. Bobinski M, de Leon MJ, Wegiel J, et al. The histological validation of post mortem magnetic resonance imagingdetermined hippocampal volume in Alzheimer's disease. Neuroscience 2000;95:721-725.

28. Johnson GA, Cofer GP, Fubara B, et al. Magnetic resonance histology for morphologic phenotyping. J Magn Reson Imaging 2002;16:423-429.
29. Benveniste H, Kim K, Zhang L, Johnson GA. Magnetic resonance microscopy of the C57BL mouse brain. Neuroimage 2000;11:601-611.

30. Helpern JA, Lee SP, Falangola MF, et al. MRI assessment of neuropathology in a transgenic mouse model of Alzheimer's disease. Magn Reson Med 2004;51:794-798.

31. Blaizot X, Martinez-Marcos A, Arroyo-Jimenez Md Mdel M, et al. The parahippocampal gyrus in the baboon: anatomical, cytoarchitectonic and magnetic resonance imaging (MRI) studies. Cereb Cortex 2004;14:231-246.

32. Benveniste H, Einstein G, Kim KR, et al. Detection of neuritic plaques in Alzheimer's disease by magnetic resonance microscopy. Proc Natl Acad Sci U S A 1999;96:14079-14084.

33. Toga AW. Brain-mapping neurotoxicity and neuropathology. Ann N Y Acad Sci 1997;820:1-13.

34. Toga AW, Thompson PM. Maps of the brain. Anat Rec 2001; 265:37-53.

35. Griffiths PD, Variend D, Evans M, et al. Postmortem MR imaging of the fetal and stillborn central nervous system. AJNR Am J Neuroradiol 2003;24:22-27.

36. Larsson EM, Englund E, Sjobeck M, et al. MRI with diffusion tensor imaging post-mortem at $3.0 \mathrm{~T}$ in a patient with frontotemporal dementia. Dement Geriatr Cogn Disord 2004;17: 316-319.

37. Schumann CM, Buonocore MH, Amaral DG. Magnetic resonance imaging of the post-mortem autistic brain. J Autism Dev Disord 2001;31:561-568.

38. Bo L, Geurts JJ, Ravid R, Barkhof F. Magnetic resonance imaging as a tool to examine the neuropathology of multiple sclerosis. Neuropathol Appl Neurobiol 2004;30:106-117.

39. Nagara $H$, Inoue $T$, Koga $T$, et al. Formalin fixed brains are useful for magnetic resonance imaging (MRI) study. J Neurol Sci 1987;81:67-77.

40. Sailer M, Fischl B, Salat D, et al. Focal thinning of the cerebral cortex in multiple sclerosis. Brain 2003;126:1734-1744.

41. Fischl B, Salat DH, Busa E, et al. Whole brain segmentation: automated labeling of neuroanatomical structures in the human brain. Neuron 2002;33:341-355.

42. Jack CR Jr, Shiung MM, Gunter JL, et al. Comparison of different MRI brain atrophy rate measures with clinical disease progression in AD. Neurology 2004;62:591-600.

43. Killiany RJ, Hyman BT, Gomez-Isla T, et al. MRI measures of entorhinal cortex vs hippocampus in preclinical AD. Neurology 2002;58:1188-1196.

44. Bernasconi N, Bernasconi A, Caramanos Z, et al. Mesial temporal damage in temporal lobe epilepsy: a volumetric MRI study of the hippocampus, amygdala and parahippocampal region. Brain 2003;126:462-469.

45. Bernasconi N, Andermann F, Arnold DL, Bernasconi A. Entorhinal cortex MRI assessment in temporal, extratemporal, and idiopathic generalized epilepsy. Epilepsia 2003;44:1070-1074.

46. Bernasconi N, Bernasconi A, Andermann F, et al. Entorhinal cortex in temporal lobe epilepsy: a quantitative MRI study. Neurology 1999;52:1870-1876.

47. Nyquist H. Certain topics in telegraph transmission theory. Trans AIEE 1928;47:617-644.

48. Ward HA. Prospective multiaxial motion correction for fMRI. Magn Reson Med 2000;43:459-469.

49. van der Kouwe A, Dale A. Real-time motion correction using octant navigators. Neuroimage 2001;13:S48. 